Rev. Biol. Trop. 52(1): 67-76, 2004

www.ucr.ac.cr www.ots.ac.cr www.ots.duke.edu

\title{
Patrones de distribución y tasas de bioerosión del erizo Centrostephanus coronatus (Diadematoida: Diadematidae), en el arrecife de Playa Blanca, Pacífico colombiano
}

\author{
Gerardo Toro-Farmer*, Jaime R. Cantera K., Edgardo Londoño-Cruz, Carlos Orozco \& \\ Raul Neira O. \\ Departamento de Biología Marina, Universidad del Valle. A.A. 25360 Cali, Colombia. \\ * getofa@hotmail.com \\ Recibido 09-III-2001. C Corregido 06-III-2002. Aceptado 24-IX-2002.
}

\begin{abstract}
Regular sea-urchins are one of the main bioeroding organisms affecting coral reefs around the world. The abundance, distribution and bioerosion rate of the sea-urchin Centrostephanus coronatus, were determined in different reef zones of Playa Blanca fringing reef (Gorgona Island, Colombian pacific coast) during 1997 and 1998. The erosion rates were determined calcinating the gut content of the sea-urchins to eliminate all organic components and preserve the inorganic portion of calcium carbonate. C. coronatus showed the highest densities towards the central zones of the reef (plain-crest and front) $\left(12.4 \mathrm{ind} / \mathrm{m}^{2}\right.$; range $\left.0-48 \mathrm{ind} / \mathrm{m}^{2}\right)$. The highest mean bioerosion rate was $0.103 \mathrm{kgCaCO}_{3} / \mathrm{m}^{2} / \mathrm{yr}$ in the reef plain-crest $\left(0-0.69 \mathrm{kgCaCO}_{3} / \mathrm{m}^{2} / \mathrm{yr}\right)$. In the other zones, (back reef and reef front) the mean bioerosion rates were 0.071 (range 0-0.39) and 0.052 (range 0-0.31) $\mathrm{kgCaCO}_{3} / \mathrm{m}^{2} / \mathrm{yr}$ respectively. According to the present data, it can be seen that the destruction of coralline skeletons, produced in this reef by sea-urchins is rather low, compared with the abrasion caused by these organisms in other places of the world. However, the combined action of $C$. coronatus and other bioeroding organisms (borers and grazers), along with some adverse environmental factors to corals, can be causing a negative balance between normal processes of reef accretion-destruction in Gorgona Island reefs.
\end{abstract}

Key words: bioerosion, sea-urchin, coral reefs, Colombia, eastern Pacific.

Aunque la bioerosión no es un fenómeno reciente en la naturaleza, actualmente se considera como uno de los procesos más destructivos para los arrecifes coralinos, ya que debilita los andamios arrecifales volviéndolos más susceptibles a cambios ambientales (cambio climático global, El Niño, etc), presiones biológicas y a la influencia antropogénica directa (e.g. turismo, contaminación, sobre-pesca) e indirecta (e.g. hipersedimentación) (Hardy et al. 1992, Grigg 1995, Jennings et al. 1995, Laroche y Ramananarivo 1995, Allison 1996, Hubbard y Parsons 1996, Acosta et al. 1996, Jiménez 2001a, 2001b, Jiménez y Cortés 2001, Vázquez-Domínguez 2003).

La bioerosión en los arrecifes coralinos puede ser realizada por dos mecanismos: la abrasión y la perforación. En el primer meca- nismo, utilizado principalmente por erizos y algunas especies de peces, el sustrato es raspado para extraer principalmente algas (filamentosas y/o calcáreas) y en algunas ocasiones, el tejido coralino; esta categoría de organismos (abrasivos) han sido considerados como los mayores agentes bioerosionadores en todo el mundo (solamente los erizos regulares son responsables de aproximadamente el $75 \%$ de la bioerosión total en el Caribe) (Bak 1994). El segundo proceso erosivo es utilizado por ciertos moluscos, sipuncúlidos, poliquetos, esponjas, etc., quienes ya sea para refugio o alimentación, perforan el esqueleto coralino debilitando su estructura. En ambos casos, la acción del hidrodinamismo marino generalmente acaba por desprender las colonias, 
ocasionando la mortalidad de los pólipos coralinos y la destrucción del andamio arrecifal.

Los organismos y los procesos que intervienen en este fenómeno han sido ampliamente estudiados en todo el mundo, especialmente en el Caribe, Indo-Pacífico y la Gran Barrera Arrecifal. En el Caribe se han estimado tasas de abrasión de $4.6 \mathrm{kgCaCO} / \mathrm{m}^{2} /$ año para el erizo Diadema antillarum (Ogden 1977); para la misma especie en Curaçao se observaron tasas de $2.9 \mathrm{~kg} / \mathrm{m}^{2} /$ año (Bak et al. 1984). En el Indo-Pacífico, Bak (1990) determinó tasas de abrasión de $4.6 \mathrm{~kg} / \mathrm{m}^{2} /$ año. En el Pacífico Oriental Tropical se han registrado valores altos de erosión por equinodermos, debido principalmente a las sobrepoblaciones de erizos después de el fenómeno de El Niño. En Panamá, después de El Niño 1982-83, las poblaciones del erizo $D$. mexicanum pasaron de $3 \mathrm{ind} / \mathrm{m}^{2}$ a $80 \mathrm{ind} / \mathrm{m}^{2}$, incrementándose también las tasas de erosión hasta 20 kgCaCO$/ \mathrm{m}^{2} /$ año (Glynn 1988). Posteriormente, para Panamá, se determinó una producción de carbonato de calcio de $14.86 \mathrm{~kg} / \mathrm{m}^{2} /$ año y una erosión total de $33.17 \mathrm{~kg} / \mathrm{m}^{2} / a n ̃ o$, de los cuales Diadema era responsable por un $5.51 \mathrm{~kg} / \mathrm{m}^{2} / \mathrm{a}-$ ño (Eakin 1996). En las Islas Galápagos, también durante El Niño 82-83, Glynn (1988) registró aumentos en las poblaciones del erizo lápiz Eucidaris thouarsii, incrementándose las tasas de erosión hasta alcanzar valores de $40 \mathrm{~kg} / \mathrm{m}^{2} /$ año. Para esta misma especie en las Islas Galápagos, Reaka-Kudla et al. (1996) determinaron tasas de material removido de 25.4 $\mathrm{kg} / \mathrm{m}^{2} / \mathrm{año}$, cifra que sigue siendo muy alta si se considera que la producción neta de carbonato de calcio en esos arrecifes no excede los $10 \mathrm{kgCaCO} / \mathrm{m}^{2} /$ año (Glynn 1988).

Para el Pacífico colombiano no se han registrado hasta el momento tasas de erosión coralina por erizos. La mayor parte de los estudios relacionados han sido enfocados a la cuantificación de las abundancias de algunos organismos coralívoros y especies asociadas al coral (Glynn et al. 1982, Rios 1987, Cantera y Contreras 1988, Guzmán y Robertson 1989, Pardo 1989, Guzmán y Lopez 1991, Cantera y Arnaud 1995, Toro-Farmer et al.
1996). Estos estudios pueden servir indirectamente para entender la magnitud del fenómeno de bioerosión puesto que existe una relación directa entre la abundancia de organismos abrasivos y las tasas de erosión en el arrecife (Bak 1994).

El principal objetivo de este estudio fue determinar los patrones de distribución y abundancia de C. coronatus, una de las especies de erizos más abundante en la Isla Gorgona y en el Pacífico colombiano, así como las tasas de erosión para individuos de diferentes tallas, utilizando la cuantificación del material calcáreo de origen coralino encontrado en su contenido estomacal. Los muestreos fueron realizados durante el evento de El Niño 1997-98, lo cual permitió comparar estos resultados con los obtenidos durante el ENSO de 1982-83.

\section{MATERIALES Y MÉTODOS}

Área de estudio: El presente estudio se realizó en el arrecife de Playa Blanca en la Isla Gorgona, Pacífico colombiano (2॰58'10’' $\mathrm{N}$ y $78^{\circ} 11^{\prime} 05^{\prime}$ 'W) (Fig. 1). Esta isla, junto con una extensa zona marina conforma un Parque Nacional Natural, con gran énfasis en la protección de las zonas coralinas. La temperatura diaria del aire en la isla fluctúa entre 27.6-30. $2^{\circ} \mathrm{C}$ y la precipitación anual alcanza valores de hasta $8000 \mathrm{~mm}$. La temperatura media anual del agua marina es de $27-28^{\circ} \mathrm{C}$ (Prahl y Erhardt 1985); la dirección predominante de las corrientes marinas en la zona es $N-N W$, alcanzando velocidades de hasta 1.5 nudos y el ciclo mareal en la isla es semidiurno, con una amplitud máxima de 4.3 m (Prahl et al. 1979).

Las zonas coralinas se encuentran principalmente en el costado oriental de la isla, el cual es protegido de la acción directa del mar abierto. En los arrecifes de la isla predominan los corales ramificados, siendo el género dominante Pocillopora spp., el cual se ha considerado como el principal constructor de estos arrecifes (Prahl et al. 1979, Glynn 2001); los corales masivos no son muy comunes $\mathrm{y}$ 


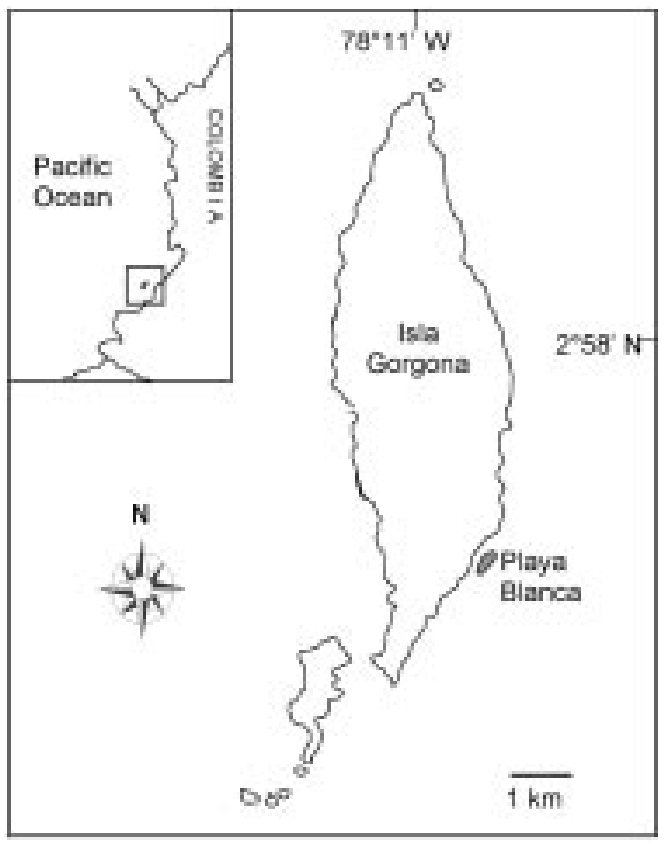

Fig. 1. Localización del arrecife de Playa Blanca en la Isla Gorgona, Pacífico colombiano.

Fig. 1. Location of Playa Blanca reef in the Gorgona Island, colombian Pacific coast.

pueden ser observados principalmente en zonas mas profundas.

El arrecife estudiado con un área aproximada de $112000 \mathrm{~m}^{2}$ fue dividido en cuatro zonas considerando, los tipos de sustratos, características topográficas y profundidad. La zona mas cercana a la playa, el Trasarrecife (TR), se caracteriza por poseer grandes parches de coral rodeados por amplias extensiones de bases coralinas despejadas (zonas de coral muerto). La Plataforma-Cresta (PC) es la de menor profundidad en el arrecife y se caracteriza por poseer las mayores coberturas de coral. En el Frente arrecifal (F) la profundidad aumenta abruptamente generando una pendiente considerable en la topografía del arrecife; en esta zona persisten los corales ramificados, aunque debido a la pendiente las colonias se desprenden facilmente cayendo hacia zonas más profundas; aparecen fragmentos de coral muerto generalmente cubiertos por al- gas calcáreas, además de grandes colonias de corales masivos. Por último la zona del Talud (TA) (la más profunda del arrecife), en donde predomina la arena, fragmentos de coral muerto, algunas pocas colonias de corales ramificados y algunas colonias masivas.

Abundancia y distribución de las especies de erizos: Los censos de erizos se realizaron en los meses de julio, septiembre y diciembre de 1997 y marzo de 1998, con la ayuda de equipo SCUBA, utilizando un total de nueve cuadrantes de $0.25 \mathrm{~m}^{2}$ ubicados al azar en cada una de las zonas, para cada fecha de muestreo; los erizos fueron buscados entre las bases de coral vivo, zonas concresionadas de coral muerto y entre fragmentos coralinos, sin remover ninguna colonia coralina ni destruir ninguna estructura arrecifal en el proceso de muestreo. Cinco ejemplares fueron llevados al laboratorio para ser comparados con especimenes de C. coronatus depositados en la Colección de referencia de Biología Marina de la Universidad del Valle (CRBMUV 84006-07); los ejemplares recolectados también fueron depositados en dicha colección (CRBMUV 2000-01).

Tasas de erosión: Por medio de observaciones directas previas, de la actividad realizada por los erizos a diferentes horas del día, se determinó que la actividad alimentaria de estos organismos es principalmente nocturna. En el mes de diciembre de 1997 fueron recolectados aproximadamente diez individuos (incluyendo ejemplares de diferentes tallas) de C. Coronatus, por zona arrecifal, durante tres períodos de tiempo: atardecer a las 1800 horas (at), media noche $\cong 0000 \mathrm{hr}(\mathrm{mn})$ y amanecer $\cong 0600 \mathrm{hr}$ (am), concordando el amanecer y el atardecer con las horas promedio de la salida y la puesta del sol en la isla. Durante los muestreos se observó que la densidad de los erizos en la zona del talud es muy baja, por lo cual en los posteriores análisis solamente fueron consideradas las otras tres zonas arrecifales. Los erizos fueron inyectados con formalina (10\%) y preservados en frascos también con formalina para ser llevados al laboratorio. Cada individuo colectado fue medido tomando el diámetro y el alto de la testa con la ayuda de un calibrador 
digital (precisión $0.01 \mathrm{~mm}$ ), en donde el diámetro fue considerado como la talla del individuo. Todos los sistemas digestivos fueron extraídos en el laboratorio y secados en una estufa a $150^{\circ} \mathrm{C}$ hasta obtener peso constante. Posteriormente, cada sistema fue calcinado en una mufla a $550^{\circ} \mathrm{C}$ durante tres horas para eliminar el material orgánico y conservar solamente la porción inorgánica de carbonato de calcio; este material remanente fue pesado en una balanza analítica (precisión $0.0001 \mathrm{~g}$ ). Los resultados obtenidos fueron extrapolados a $\mathrm{kg}$ $\mathrm{CaCO}_{3} / \mathrm{m}^{2} /$ año utilizando la densidad de erizos promedio por zona en $0.25 \mathrm{~m}^{2}$ y un día de tiempo. Para éstos análisis se consideró que el material existente en el contenido estomacal corresponde a un día de alimentación (Glynn 1988, Conand et al. 1996).

\section{RESULTADOS}

Densidad, distribución espacial y temporal: C. coronatus presentó una densidad pro- medio de $6.86 \mathrm{ind} / \mathrm{m}^{2}$ y un número máximo de $48 \mathrm{ind} / \mathrm{m}^{2}$, mostrando diferencias significativas de su abundancia entre las zonas arrecifales (ANOVA Factorial, $\mathrm{p}<0.001$ ). Las mayores densidades fueron observadas hacia las zonas centrales (plataforma-cresta y frente arrecifal) y las menores hacia las zonas marginales del arrecife (trasarrecife y talud) (Fig. 2).

No se presentaron diferencias significativas de la abundancia de $C$. coronatus entre los períodos de estudio (ANOVA Factorial, p>0.001) (Fig. 2).

Tallas de erizos, zonas arrecifales y períodos de alimentación, como determinantes de las tasas de erosión: Se establecieron cuatro rangos de tallas o categorías, a partir de los diámetros de las testas obtenidos de los individuos colectados y de las observaciones realizadas en el arrecife: a) tallas pequeñas, con diámetros inferiores a $1.0 \mathrm{~cm}$; b) tallas intermedias, entre 1.0 y $1.5 \mathrm{~cm}$, las cuales son las más frecuentes en todo el arrecife, principalmente en la zona PC; c) mayores de 1.5 y menores de $2.0 \mathrm{~cm}$; y d) tallas grandes, con más
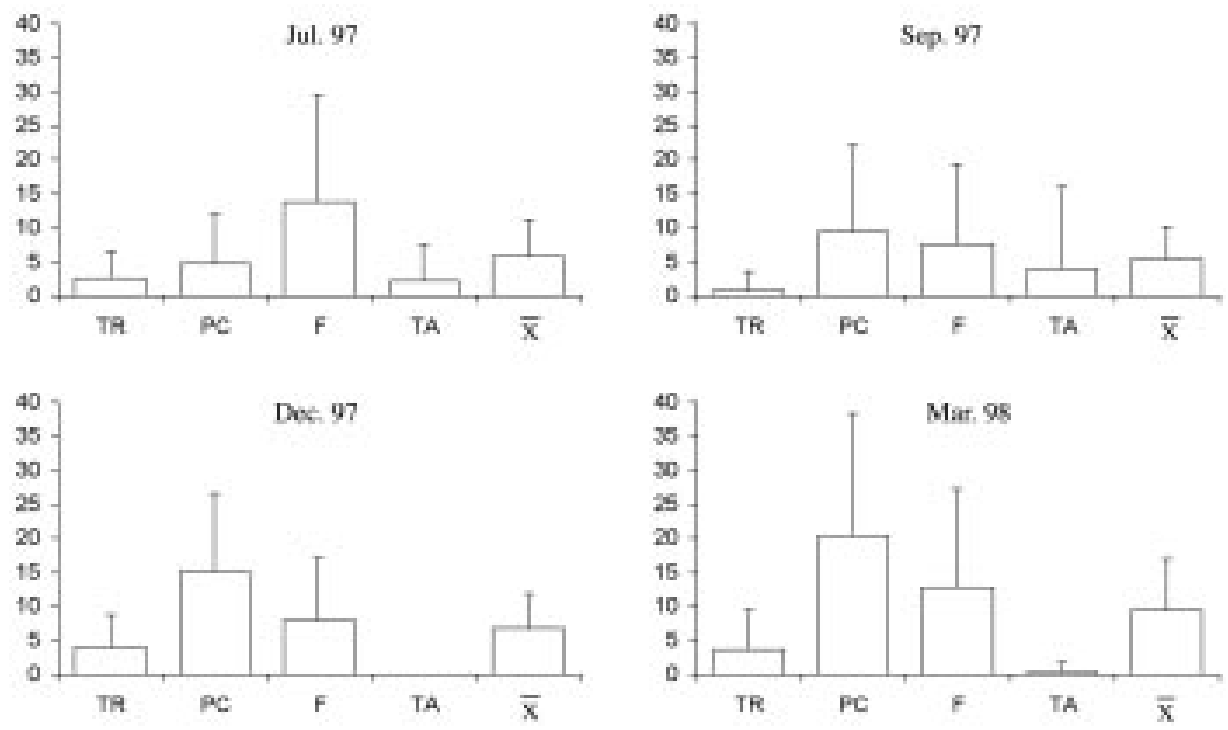

Fig. 2. Densidad a través del tiempo de estudio de C. coronatus para cada una de las zonas arrecifales en Playa Blanca y densidad promedio $( \pm$ D.E. $)$. $\mathrm{TR}=$ trasarrecife $\mathrm{PC}=$ plataforma-cresta; $\mathrm{F}=$ frente; $\mathrm{TA}=$ talud.

Fig. 2. Density during the study period of $C$. coronatus in Playa Blanca reef zones and mean density $( \pm \mathrm{S} . \mathrm{D}$.). TR= back reef.; $\mathrm{PC}=$ reef plain-crest; $\mathrm{F}=$ front reef; $\mathrm{TA}=$ reef slope. 
de $2.0 \mathrm{~cm}$ de diámetro. Entre los individuos recolectados más de la mitad pertenecen a la segunda categoría (Fig. 3).

Se presentaron diferencias significativas de las tasas de erosión entre las cuatro categorías de tallas (ANOVA, $\mathrm{p}<0.05, \mathrm{n}=94$ ), mostrando las tasas mas altas de bioerosion para los erizos mas grandes (Cuadro 1). Sin embargo, las tasas de erosión mostraron una correlación muy baja pero significativa con el diámetro (Pearson, $\mathrm{r}^{2}=0.22 ; \mathrm{p}<0.005$; Tasa $=$ 0.0504+0.08467 diámetro).

Las tasas de erosión promedio también presentaron diferencias significativas entre las zonas arrecifales (ANOVA Factorial, $\mathrm{p}<0.001$ ) y la plataforma-cresta mostró la tasa promedio mas alta (Cuadro 1). Entre los tres períodos de alimentación (atardecer, media noche y amanecer) no se presentaron diferencias significativas en las tasas de erosión (ANOVA Factorial, $\mathrm{p}>0.05$ ).

Erosión promedio y tasas máximas de erosión en el arrecife: La tasa de erosión promedio para el arrecife fue de $0.19 \mathrm{kgCaC}-$ $0_{3} / \mathrm{m}^{2} / \mathrm{año}$, la cual se calculó utilizando la densidad media encontrada de erizos $(6.86$ $\mathrm{ind} / \mathrm{m}^{2}$ ) y el peso promedio de material calcáreo presente en los individuos analizados (0.075 g/ind/día; $\mathrm{n}=94)$. La mayor tasa de erosión calculada se presentó en el trasarrecife con un valor de $1.41 \mathrm{kgCaCO}_{3} / \mathrm{m}^{2} /$ año, a partir

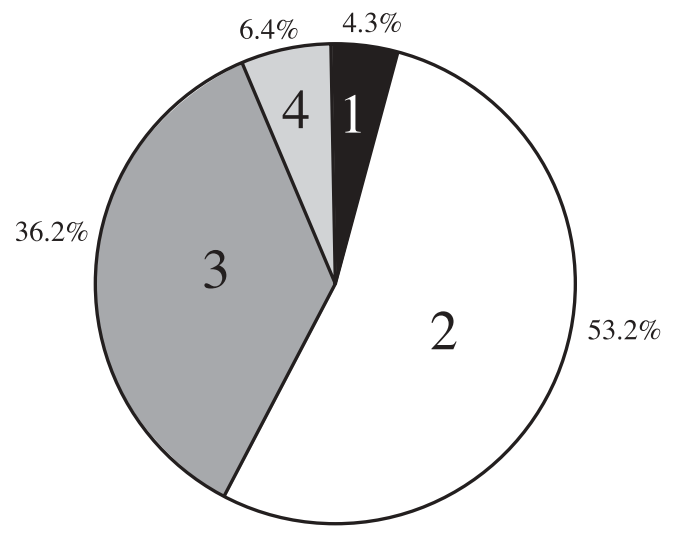

Fig. 3. Porcentaje de individuos para cada categoría de talla de $C$. coronatus. A: categoría $1(<1.0 \mathrm{~cm})$; B: categoría 2 (1.0-1.5 cm); C: categoría $3(1.5-2.0 \mathrm{~cm})$; D: categoría 4 $(>2.0 \mathrm{~cm})$.

Fig. 3. Size categories percents for $C$. coronatus. A: category $1(<1.0 \mathrm{~cm})$; B: category $2(1.0-1.5 \mathrm{~cm})$; C: category $3(1.5-2.0 \mathrm{~cm})$; D: category $4(>2.0 \mathrm{~cm})$.

de una densidad máxima de $48 \mathrm{ind} / \mathrm{m}^{2}$ y un peso máximo de $0.0803 \mathrm{~g} / \mathrm{ind} /$ día. Para las otras zonas, las mayores tasas fueron de $0.75 \mathrm{~kg}$ $\mathrm{CaCO}_{3} / \mathrm{m}^{2} /$ año en la plataforma-cresta y 0.51 $\mathrm{kgCaCO} 3_{3} / \mathrm{m}^{2} /$ año en el frente arrecifal, ambos valores calculados utilizando también la mayor densidad registrada y el mayor peso de material calcáreo encontrado en cada zona.

CUADRO 1

Tallas de erizos y zonas arrecifales como determinantes de las tasas de erosión ( $\mathrm{kg} / \mathrm{m} 2 / a \tilde{n} o \pm D E)$

TABLE 1

Size of sea-urchins, reef zones and erosion rates $(\mathrm{kg} / \mathrm{m} 2 / \mathrm{yr} \pm \mathrm{SD})$

Categoría de Talla

$<1.0 \mathrm{~cm}$

$1.0-1.5 \mathrm{~cm}$

$1.5-2.0 \mathrm{~cm}$

$>2.0 \mathrm{~cm}$

Zonas arrecifales

Trasarrecife

Plataforma-cresta

Frente
Número de individuos (n)

4

50

34

6

31

32

31
Tasas de erosión kg/m²/año ( $\pm \mathrm{DE})$

$$
\begin{aligned}
& 0.034 \pm 0.044 \\
& 0.058 \pm 0.092 \\
& 0.101 \pm 0.174 \\
& 0.110 \pm 0.131
\end{aligned}
$$

$0.071 \pm 0.029$

$0.103 \pm 0.056$

$0.052 \pm 0.063$ 


\section{DISCUSIÓN}

Las densidades de $C$. coronatus encontradas en el arrecife estudiado son relativamente altas, comparables solamente con otras localidades en donde, factores ambientales muy específicos, limitan las poblaciones de algunas especies de erizos favoreciendo a unas pocas especies dominantes. Algunos de estos factores en el Caribe e Indo-Pacífico son la sobrepesca de especies competidoras o depredadoras de erizos, la contaminación de las aguas donde habitan las poblaciones de erizos y la contaminación orgánica que favorece el crecimiento de algas (Ogden 1977, Bak 1990, Conand et al. 1996, Glynn 1997). En estos casos, el aumento en la densidad de algunas especies de erizos se ha traducido en un aumento de las tasas de erosión ocasionadas por estos organismos, causando mayor destrucción en los arrecifes.

Las altas temperaturas del mar en el Pacífico oriental tropical asociadas a El Niño, causan altas mortandades de corales, favoreciendo casi siempre el rápido crecimiento de algas sobre los esqueletos de coral muerto y por consiguiente, un aumento en la abundancia de erizos y en sus tasas de bioerosión (Glynn 1988, Eakin 1992, Reaka-Kudla et al. 1996, Glynn 1997). Durante los primeros meses de estudio (julio 1997) se presentó la anomalía térmica de El Niño y el agua marina en el arrecife alcanzó temperaturas de hasta $32^{\circ} \mathrm{C}$ en diciembre del 97. Como consecuencia de estas altas temperaturas, sumado a las prolongadas exposiciones aéreas y solares durante las mareas bajas extremas, amplias zonas de coral fueron blanqueadas y parches de algas filamentosas empezaron a crecer sobre ellos. Sin embargo, hubo una rápida recuperación coralina y al cabo de cuatro meses disminuyeron nuevamente las poblaciones de algas. A pesar de que no se presentaron diferencias significativas en la abundancia de $C$. coronatus entre los períodos de estudio, se pudo observar cómo la abundancia de esta especie aumentó levemente después del evento climático, mostrando sus mayores densidades en el mes de marzo del 98, justamente después del calentamiento en el 97.
Densidades altas de erizos también fueron registradas en los arrecifes de la isla durante 1996 (Toro-Farmer et al. 1996), año en el cual no se presentaron anomalías climáticas asociadas. Sin embargo, aumentos temporales en las densidades de erizos pueden ser frecuentes en Gorgona ya que sus arrecifes durante años anteriores también han sido fuertemente afectados por perturbaciones ambientales como El Niño (Prahl 1985, Glynn 1990), prolongadas exposiciones aéreas (Zapata et al. 1996) y épocas de hipersedimentación con materiales provenientes de la costa o del borde insular (obs. pers.). Grandes extensiones de coral muerto y rápido crecimiento de algas filamentosas sobre estos nuevos sustratos disponibles han sido observados después de ocurridos estos fenómenos.

La principal fuente de alimento para algunos erizos son las algas verdes, que crecen sobre el esqueleto coralino en las partes que quedan desprovistas de pólipos. Sin embargo, en numerosos estudios se ha demostrado la presencia de carbonato de calcio, proveniente de algas calcáreas y de coral, en el contenido estomacal de estos organismos bioerosionadores (Glynn 1988, Eakin 1992, Conand et al. 1996, Glynn 1997, James 2000), lo cual indica que de una u otra forma, algunas especies de erizos al raspar el sustrato en busca de su alimento, también puede abrasionar estructuras coralinas en donde crecen las algas calcáreas. A diferencia de algunas especies de erizos cuya alimentación se basa principalmente en álgas calcáreas (James 2000), C. coronatus no incluye este tipo de algas dentro de su dieta, convirtiéndose su ingestión en algo accidental y mostrando que las similitudes encontradas, entre el patrón de distribución de esta especie y el de las algas calcáreas (en las bases coralinas y grietas en el sustrato), no son debidas a predación sobre estas algas, sino justamente porque el erizo las "excluye" de su dieta permitiendo su crecimiento (Vance 1979).

Las tasas de erosión presentan una relación directa con la abundancia de los organismos abrasivos (Bak 1994). En la zona arrecifal donde la densidad es mayor (plataforma-cresta), la tasa de erosión también fue mayor. Este 


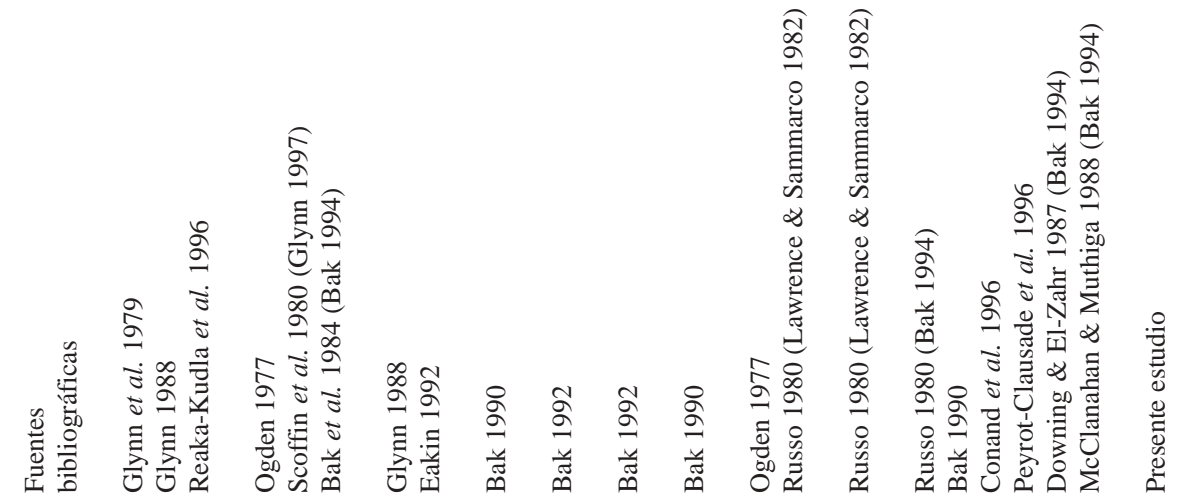

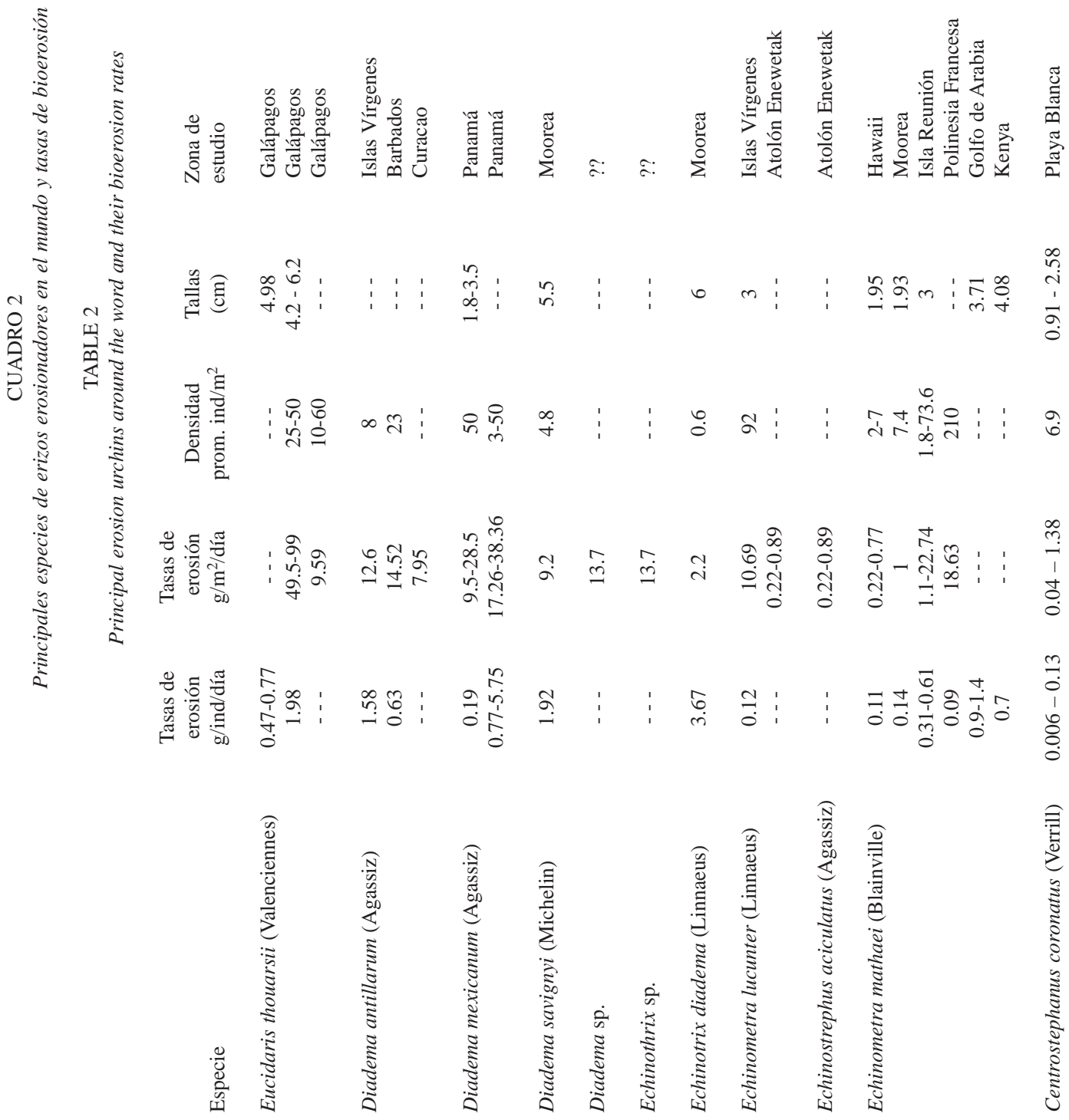


hecho demuestra el balance que existe entre construcción y destrucción arrecifal, ya que esta zona a pesar de ser la que ha sufrido mayor erosión, también presenta las mayores coberturas de coral vivo finalmente determinadas por el crecimiento arrecifal. En los arrecifes de Gorgona han sido registradas hasta el momento cinco especies de erizos (Pardo 1989, ToroFarmer et al. 1996, Toro-Farmer 1998), incluyendo especies que se consideran potencialmente erosionadoras en abundancias altas (Familias Diadematidae, Cidaridae y Toxopneustidae); por este motivo, se podría plantear la hipótesis de que las tasas netas de abrasión para el arrecife, considerando a otras especies bioerosionadoras, son más altas que las encontradas en el presente estudio que solamente incluye a $C$. coronatus. Sin embargo en general, durante todo el período de estudio, las densidades encontradas para las otras especies fueron muy bajas, casi siempre por debajo de 1 ind $/ \mathrm{m}^{2}$.

Otro de los factores que determinan las tasas de erosión por erizos, es la talla o tamaño de los individuos implicados en el proceso (Bak 1994, Conand et al. 1996). La talla de los erizos en Playa Blanca es pequeña, comparada con la de individuos encontrados en diferentes sitios a nivel mundial (Cuadro 2). Se observó que las tasas de erosión en Playa Blanca aumentan cuando aumenta el tamaño de $C$. Coronatus, presentándose variaciones significativas entre tallas. Es de suponer entonces, que éste sea uno de los principales factores que determina las tasas de erosión en el arrecife, junto con las variaciones en la densidad de los erizos. Tallas similares en diferentes sitios han mostrado tasas de erosión cercanas a las encontradas en el presente estudio (Glynn 1988, Conand et al. 1996); en estos lugares, pequeñas fluctuaciones en el tamaño promedio de las especies de erizos, se han reflejado en cambios fuertes de las tasas de erosión, lo cual puede sugerir que en períodos de tiempo, en los cuales las tallas aumenten, el impacto de los erizos sobre la estructura arrecifal en Gorgona puede ser más alto.

Se concluye que las tasas de erosión por erizos en el arrecife de Playa Blanca son bajas, comparadas con las tasas encontradas en otros sitios del mundo. Se necesitan más datos sobre crecimiento coralino en la isla para poder determinar si el proceso crecimiento-destrucción se encuentra balanceado o por el contrario, uno de los procesos supera cuantitativamente al otro. Sin embargo, se puede asumir que bajo condiciones ambientales adversas para el crecimiento arrecifal (El Niño y sus altas temperaturas, períodos prolongados de exposición aérea durante mareas bajas extremas, hipersedimentación, etc.), las tasas de erosión por erizos, sumadas a los efectos de otros organismos, pueden desequilibrar considerablemente el balance entre construcción y destrucción arrecifal.

\section{AGRADECIMIENTOS}

Este estudio se realizó como parte del proyecto "Relación entre las tasas de bioerosión y crecimiento en arrecifes coralinos de la 1sla Gorgona, Pacífico colombiano" financiado por Colciencias-Colombia (1106-09-06996) y apoyado por la Universidad del Valle y la Unidad de Parques Nacionales Naturales. Agradecemos a I. Romero, E. Peña y G. Bolívar por las revisiones del manuscrito y a P. Forero por su colaboración en el trabajo de campo. También a C. Acevedo y G. Mayor por su colaboración permanente e incondicional en el Parque.

\section{RESUMEN}

Los erizos regulares son uno de los principales grupos de organismos bioerosionadores que destruyen los arrecifes coralinos alrededor del mundo. En el presente estudio se determinaron los patrones de distribución y abundancia y las tasas de carbonato de calcio removido en el arrecife de Playa Blanca por Centrostephanus coronatus, una de las especies de erizos más abundante en este arrecife. Para este fin se realizaron cuatro visitas al arrecife entre 1997 y 1998 durante las cuales se contabilizó el número de individuos en cada una de las principales zonas arrecifales (Trasarrecife, Plataforma-Cresta, Frente, y Talud). Las tasas de erosión fueron determinadas por medio del análisis del contenido estomacal de un número 
representativo de individuos colectados en cada zona. Cada sistema digestivo fue calcinado para eliminar la materia orgánica y conservar solamente la porción inorgánica de carbonato de calcio. C. coronatus presentó sus mayores densidades hacia las zonas centrales del arrecife (12.4 ind $/ \mathrm{m}^{2}$; rango 0-48 ind $/ \mathrm{m}^{2}$ ). La tasa de bioerosión promedio más alta de $C$. coronatus fue de $0.103 \mathrm{kgCaCo}-$ ${ }_{3} / \mathrm{m}^{2} /$ año en la Plataforma-Cresta (rango 0 a $0.69 \mathrm{kgCaCo}-$ ${ }_{3} / \mathrm{m}^{2} / \mathrm{año}$ ). En las otras zonas (trasarrecife y frente) las tasas promedio de erosión fueron $0.071(0-0.39)$ y 0.052 $(0-0.31) \mathrm{kgCaCo}_{3} / \mathrm{m}^{2} /$ año respectivamente. De acuerdo con estos datos fue posible comprobar que la destrucción de esqueletos de corales en este arrecife por esta especie de erizo es baja, comparada con la acción abrasionadora de este tipo de organismos en otras partes del mundo. Sin embargo, la acción combinada de $C$. coronatus con otros organismos bioerosionadores y con algunos factores ambientales adversos para los corales, puede estar provocando un desbalance entre los procesos normales de contrucción-destrucción arrecifal en la Isla Gorgona.

\section{REFERENCIAS}

Acosta, A., S. Fowler, J.L.Teyssie \& J. Jaubert. 1996. Uptake and release of heavy metals and radionuclides in corals. Abstracts, 8th Int. Coral Reef Symp., Panamá.

Allison, W.R. 1996. Snorkeler damage to reef corals in the Maldive Islands. Coral Reefs 15: 215-218.

Bak, R.P.M. 1990. Patterns of echinoid bioerosion in two Pacific coral reefs lagoons. Mar. Ecol. Prog. Ser. 66: 267-272.

Bak, R.P.M. 1992. Echinoids and their impact on coral reef carbonate budgets in past, present and future. Abstracts, 7th Int. Coral Reef Symp.

Bak, R.P.M. 1994. Sea urchin bioerosion on coral reefs: place in the carbonate budget and relevant variables. Coral Reefs 13: 99-103.

Bak, R.P.M., M.J.E. Carpay \& E.D. De Ruyter van Steveninck. 1984. Densities of the sea urchin Diadema antillarum before and after mass mortalities on the coral reefs of Curaçao. Mar. Ecol. Prog. Ser. 17: 105108.

Cantera, J.R. \& R. Contreras. 1988. Bivalvos perforadores de esqueletos de corales escleractiniarios en la Isla de Gorgona, Pacífico Colombiano. Rev. Biol. Trop. 36: $151-158$.

Cantera, J.R. \& P.M. Arnaud. 1995. Coral reef associated molluscan assemblages in Gorgona island, Colombian Pacific coast. Haliotis 24: 87-100.
Conand, C., P. Chabanet, P. Cuet \& Y. Letourneur. 1996. The carbonate budget of a fringing reef in La Reunion Island (Indian ocean): external bioerosion and benthic flux of $\mathrm{CaCO}_{3}$. Abstracts, 8th Int. Coral Reef Symp., Panamá.

Eakin, C.M. 1992. Post-El Niño panamanian reefs: less accretion, more erosion and Damselfish protection. Proc. 7th Int. Coral Reefs Symp. 1: 387-396.

Eakin, C.M. 1996. Where have all the carbonates gone? A model comparison of calcium carbonate budgets before and after the 1982-1983 El Niño at Uva Island in the eastern Pacific. Coral Reefs 15: 109-119.

Glynn, P.W. 1988. El Niño warming, coral mortality and reef framework destruction by echinoid bioerosion in the eastern pacific. Galaxea 7: 129-160.

Glynn, P.W. 1990. Coral mortality and disturbances to coral reefs in the tropical eastern Pacific, pp. 55-126. In Glynn P.W. (ed.) Global ecological consequences of the 1982-83 El Niño-Southern Oscillation. Elsevier, Amsterdam.

Glynn, P.W. 1997. Bioerosion and coral-reef growth: a dynamic balance, pp. 68-95. In C. Birkeland (ed.). Life and Death of Coral Reefs. Chapman \& Hall, New York.

Glynn, P.W. 2001. Eastern Pacific coral reef ecosystems, pp. 281-305. In Seeliger U. \& B. Kjerfve (eds.). Coastal Marine Ecosystems of Latin America, Ecological studies v. 144. Springer-Verlag, New York.

Glynn, P.W., G.M. Wellington \& C. Birkeland. 1979. Coral reef growth in the Galápagos: limitation by sea urchins. Science 203: 47-49.

Glynn, P.W., H. Prahl \& F. Guhl. 1982. Coral reefs of Gorgona Island, Colombia, with special reference to corallivores and their influence on community structure and reef development. An. Inst. Inv. Mar. Punta de Betín 12: 185-214.

Grigg, R.W. 1995. Coral reefs in an urban embayment in Hawaii: a complex case history controlled by natural and anthropogenic stress. Coral Reefs 14: 253-266.

Guzmán, H.M. \& D.R. Robertson. 1989. Population and feeding responses of the corallivorous pufferfish Arothron meleagris to coral mortality in the eastern Pacific. Mar. Ecol. Prog. Ser. 55: 121-131.

Guzmán, H.M. \& J.D. Lopez. 1991. Diet of the corallivorous pufferfish Arothron meleagris (Pisces: Tetraodontidae) at Gorgona Island, Colombia. Rev. Biol. Trop. 39: 203-206.

Hardy, J.T., F.E. Hoge, J.K. Yungel \& R.E. Dodge. 1992. Remote detection of coral 'bleaching' using pulsed-laser 
fluorescence spectroscopy. Mar. Ecol. Prog. Ser. 88: 247-255.

Hubbard, D.K. \& K.M. Parsons. 1996. Dredging, reef destruction and beach erosion: a case study. Abstracts, 8th Int. Coral Reef Symp., Panamá.

James, D.W. 2000. Diet, movement, and covering behavior of the sea urchin Toxopneustes roseus in rhodolith beds in the Gulf of California, México. Mar. Biol. 137: 913-923.

Jennings, S., E.M. Grandcourt \& N.V.C. Polunin. 1995. The effects of fishing on the diversity, biomass and trophic structure of Seychelles' reef fish communities. Coral Reefs 14: 225-235.

Jiménez, C. 2001a. Arrecifes coralinos de Bahía Culebra, Pacífico de Costa Rica: aspectos biológicos, económico-recreativos y de manejo. Rev. Biol. Trop. 49 (Supl. 2): 215-231.

Jiménez, C. 2001b. Beaching and mortality of ref. organism during a warming event in 1995 on the Caribbean coast of Costa Rica. Rev. Biol. Trop. 49 (Supl. 2): 233-238.

Jiménez, C. \& J.Cortés. 2001. Effects of the 1991-1992 El Niño on scleractiniam corals of the Costa Rican central Pacific coast. Rev. Biol. Trop. 49 (Supl. 2): 239-250.

Laroche, J. \& N. Ramananarivo. 1995. A preliminary survey of the artisanal fishery on coral reefs of the Tulear Region (southwest Madagascar). Coral Reefs 14: $193-200$.

Lawrence, J.M. \& P.W. Sammarco. 1982. Effects of feeding on the environment: Echinoidea. In M. Jangoux \& J.M. Lawrence (eds). Echinoderm nutrition. A.A. Balkema, Rotterdam.

Ogden, J.C. 1977. Carbonate-sediment production by parrot fish and sea urchins on Caribbean reefs, pp. 281288. In S.H. Frost, M.P. Weiss \& J.B. Saunders (eds.). Reef and related carbonates-ecology and sedimentology. American Association of Petroleum Geologists, Tulsa.

Pardo, R. 1989. Estudio taxonómico y ecológico de los principales grupos de equinodermos del litoral Pacífico colombiano. Tesis de grado, Universidad del Valle, Cali, Colombia.
Peyrot-Clausade, M., N. Pari, P. Hutchings, T. Le Campion-Alsumard, V. Chazottes \& M.F. Fontaine. 1996. Bioerosion and bioaccretion balance: two years of experimental studies. Abstracts, 8th Int. Coral Reef Symp., Panamá.

Prahl, H. 1985. Blanqueo masivo y muerte de corales hermatípicos en el Pacífico colombiano atribuidos al Fenómeno de El Niño 1982-83. Boletín ERFEN 12: 22-24.

Prahl, H. \& H. Erhardt. 1985. Colombia: Corales y Arrecifes coralinos. FEN, Bogotá. 295 p.

Prahl, H, F. Guhl \& M. Grogl. 1979. Gorgona. Futura. Bogotá. 279 p.

Reaka-Kudla, M.L., J.S. Feingold \& W. Glynn. 1996. Experimental studies of rapid bioerosion of coral reefs in the Galápagos Islands. Coral Reefs 15: 101-107.

Rios, R. 1987. La fauna asociada al coral vivo Pocillopora damicornis en la Isla Gorgona, Pacífico colombiano. Rev. Asoc. Colomb. Cien. Biol. 4: 22-26.

Toro-Farmer, G. 1998. Estimación de la intensidad bioerosionadora de los erizos (Echinodermata) en un arrecife coralino de la Isla Gorgona, Pacífico colombiano. Tesis de grado, Universidad del Valle, Cali, Colombia.

Toro-Farmer, G., I.C. Romero, J.R. Cantera K, R. Neira \& E. Rodriguez. 1996. Distribución de erizos y cobertura de sustratos en el arrecife de la azufrada (Isla Gorgona): comparación entre cuadrantes in situ y cuadrantes fotográficos. X Seminario Nacional de Ciencias y Tecnologías del Mar, Bogotá.

Vance, R.R. 1979. Effects of grazing by the sea-urchin, Centrostephanus coronatus, on prey Community Composition. Ecology 60 (3): 537-546.

Vázquez-Domínguez, E. 2003. Diversidad y distribución de crustáceos y equinodermos y su relación con niveles de sedimentación en arrecifes coralinos. Rev. Biol. Trop. 51: 183-194.

Zapata, F.A., J.M. Jimenez, C.E. Barcenas \& R. Neira. 1996. Frecuencia e impacto de eventos de exposición aérea durante mareas bajas extremas en un arrecife coralino de Gorgona. Resúmenes X Seminario Nacional de Ciencias y Tecnologías del Mar, Bogotá. 